\title{
Production of glycolipid biosurfactants, mannosylerythritol lipids, from pentoses and D-glucose/D-xylose mixtures by Pseudozyma yeast strains
}

\author{
Nuno Torres Faria ${ }^{a, b, c}$, Marisa V. Santos ${ }^{a}$, Pedro Fernandes ${ }^{a, d}$, Luís Lopes Fonseca ${ }^{e, 1}$, \\ César Fonseca ${ }^{\mathrm{c}, *}$, Frederico Castelo Ferreira ${ }^{\mathrm{a}, \mathrm{b}, * *}$ \\ a Department of Bioengineering and IBB-Institute for Biotechnology and Bioengineering, Instituto Superior Técnico, Universidade de Lisboa, \\ Av. Rovisco Pais, 1049-001 Lisboa, Portugal \\ ${ }^{b}$ MIT-Portugal Program, 77 Massachusetts Avenue, E40-221, Cambridge, MA 02139, USA \\ c Laboratório Nacional de Energia e Geologia, I.P., Unidade de Bioenergia, Estrada do Paço do Lumiar 22, 1649-038 Lisboa, Portugal \\ d Faculdade de Engenharia, Universidade Lusófona de Humanidades e Tecnologias, Av. Campo Grande 376, 1749-024 Lisboa, Portugal \\ e Instituto de Tecnologia Química e Biológica (ITQB), Universidade Nova de Lisboa, 2780-156 Oeiras, Portugal
}

\section{A R T I C L E I N F O}

\section{Article history:}

Received 24 June 2014

Received in revised form 28 July 2014

Accepted 14 August 2014

Available online 23 August 2014

\section{Keywords:}

Manosylerithritol lipids (MEL)

Biosurfactants

Pentoses

D-Xylose

Pseudozyma spp.

\begin{abstract}
A B S T R A C T
The pentose-assimilating capacity of yeasts from the genus Pseudozyma, $P$. antarctica PYCC $5048^{\mathrm{T}}, P$. aphidis PYCC $5535^{\mathrm{T}}$ and $P$. rugulosa PYCC $5537^{\mathrm{T}}$, was exploited towards the production of mannosylerythritol lipids (MEL), a glycolipid with biosurfactant properties. The three strains tested were able to grow on D-xylose and L-arabinose with similar maximum specific growth rates to those estimated on D-glucose (around $0.2 \mathrm{~h}^{-1}$ ). The highest MEL titres $(4.8-5.4 \mathrm{~g} / \mathrm{l})$ and yields $(0.11-0.14 \mathrm{~g} / \mathrm{g}$ ) from D-xylose were found in P. antarctica PYCC 5048 ${ }^{\mathrm{T}}$, which presented similar values to those estimated on D-glucose and on D-xylose/D-glucose mixtures. P. rugulosa PYCC $5537^{\mathrm{T}}$ showed a pattern of sugar conversion into MEL similar to $P$. antarctica, but at $40 \%$ lower titres. P. aphidis PYCC $5535^{\mathrm{T}}$ presented lower MEL titres from $\mathrm{D}$-xylose $(1.2 \mathrm{~g} / \mathrm{l})$ than from $\mathrm{D}$-glucose $(3.4 \mathrm{~g} / \mathrm{l})$. Nitrate supply increased sugar consumption rate and, when accompanied by D-glucose or D-xylose feeding, additional biomass production. In this case, sugar was completely consumed before sugar feeding at day 7 , but not when feeding is performed at day 4. Higher MEL titres where obtained for the later condition reaching $7.3 \mathrm{~g} / \mathrm{l}$ and $5.8 \mathrm{~g} / \mathrm{l}$, in fed-batch cultures with glucose and xylose, respectively.
\end{abstract}

(c) 2014 Elsevier Ltd. All rights reserved.

\section{Introduction}

Microbial surfactants are recognized as fine chemicals with high-value applications and interesting characteristics such as low toxicity, high biodegradability, effectiveness at extreme temperatures or $\mathrm{pH}$, and mild production conditions when compared to

\footnotetext{
* Corresponding author at: Laboratório Nacional de Energia e Geologia, I.P., Unidade de Bioenergia, Estrada do Paço do Lumiar 22, 1649-038 Lisboa, Portugal. Tel.: +351210924717

** Corresponding author at: Department of Bioengineering and IBB-Institute for Biotechnology and Bioengineering, Instituto Superior Técnico, Universidade de Lisboa, Av. Rovisco Pais, 1049-001 Lisboa, Portugal. Tel.: +351 218419598.

E-mail addresses: cesar.fonseca@lneg.pt (C. Fonseca), frederico.ferreira@ist.utl.pt, frederico_castelo@yahoo.com (F.C. Ferreira).

1 Present address: Integrative BioSystems Institute and The Wallace H. Coulter Department of Biomedical Engineering, Georgia Institute of Technology, 313 Ferst Drive, Atlanta, GA 30332, USA.
}

chemical surfactants [1-3]. Biosurfactants, in particular lipopeptides and glycolipids, have industrial applications in the production of food, cosmetics, and pharmaceutics, as well as for soil decontamination of heavy metals, oils and other toxic organics [4]. In microbial environments, the roles of biosurfactants include the increase of surface area and bioavailability of hydrophobic waterinsoluble substrates, heavy metal binding, bacterial pathogenesis, quorum sensing and biofilm formation [2].

Mannosylerythritol lipids (MELs) are glycolipids containing a 4-O- $\beta$-D-mannopyranosyl-meso-erythritol as the glycosidic/hydrophilic moiety and two short-chain fatty acids (usually C8-C12) as the hydrophobic groups (Fig. 1) [4]. These amphiphilic properties classify these molecules as biosurfactants [1]. MELs are designated as MEL-A, -B, -C, according with their elution position in thin layer chromatography (TLC), and corresponding to the degree of acetylation at C-4 and/or at C- 6 position of the mannosyl moiety [5]. MEL-A corresponds to the diacetylated compound while MEL-B and MEL-C are monoacetylated at C- 6 and C-4 of 